University of Nebraska - Lincoln

DigitalCommons@University of Nebraska - Lincoln

Faculty Publications from the Department of Engineering Mechanics

Mechanical \& Materials Engineering,

Department of

2008

\title{
On Constitutive Models for Limited Elastic, Molecular Based \\ Materials
}

Millard F. Beatty

University of Nebraska - Lincoln, mbeatty2@unl.edu

Follow this and additional works at: https://digitalcommons.unl.edu/engineeringmechanicsfacpub

Part of the Mechanical Engineering Commons

Beatty, Millard F., "On Constitutive Models for Limited Elastic, Molecular Based Materials" (2008). Faculty Publications from the Department of Engineering Mechanics. 59.

https://digitalcommons.unl.edu/engineeringmechanicsfacpub/59

This Article is brought to you for free and open access by the Mechanical \& Materials Engineering, Department of at DigitalCommons@University of Nebraska - Lincoln. It has been accepted for inclusion in Faculty Publications from the Department of Engineering Mechanics by an authorized administrator of DigitalCommons@University of Nebraska - Lincoln. 
Published in Mathematics and Mechanics of Solids 13 (2008), pp. 375-387; doi

10.1177/1081286507076405 Copyright @ 2007 Sage Publications. Used by permission.

http://mms.sagepub.com/cgi/content/abstract/13/5/375

Submitted November 3, 2006; accepted December 13, 2006; published online May 14, 2007.

\title{
On Constitutive Models for Limited Elastic, Molecular Based Materials
}

\author{
Millard F. Beatty \\ Department of Engineering Mechanics, University of Nebraska-Lincoln \\ Dedicated to Professor Cornelius O. Horgan in esteem and friendship, with highest regard.
}

\begin{abstract}
:
The response function for a general class of elastic molecular based materials characterized by their limiting molecular chain extensibility and depending on only the first principal invariant of the Cauchy-Green deformation tensor together with a certain molecular based limiting extensibility parameter is introduced. The constitutive response function for the Gent material is then derived inversely as the [0/1] Padé approximant of this class, a result that leads naturally to an infinite geometric series representation of its response function. Truncation of this series function characterizes a familiar class of quadratic materials now having physically relevant material constants. It is shown that the [0/2] approximant of the response function for the general class of restricted elastic materials leads inversely to a new constitutive model and its series representation. Of course, many familiar limited elastic material models are members of the general class. The Padé approximants for some response functions are not, and empirical modifications that admit these as members of the general class are described. Examples of two limited elastic models in the class that are not Padé approximants are noted. The strain energy functions for a few of the restricted elastic models described are presented.
\end{abstract}

Keywords: Constitutive models, rubber elasticity, molecular based materials, limiting chain extensibility, Padé approximants, Gent model, Arruda-Boyce model, strain energy functions

\section{Introduction}

The classical non-Gaussian statistical theory of molecular based rubberlike materials [1, 2] long ago established the limited elasticity of even the most highly elastic materials due to the finite extensibility of their molecular chains. As a consequence, the stress response of the material in a simple extension, for example, grows indefinitely great as the stretch approaches its ultimate value imposed by the limiting molecular chain extensibility in an affine deformation of the material. Experiments by Dickie and Smith [3] in simple extension, pure shear and equibiaxial deformation show that the degree of limiting extensibility of the material, however, varies with the type of deformation. The limiting extensibility in a pure shear is only slightly smaller, whereas in an equibiaxial 
extension it is generally about $30 \%$ smaller than that in a simple extension. These differences, however, are resolved by their adopting a limiting value of the first principal invariant as a deformation invariant measure of the limited extensibility of the material. This is consistent with the results of basic non-Gaussian molecular based constitutive theory. The fundamental molecular theory, however, leads to a formidable constitutive relation for the general mechanical response of the material. To circumvent this difficulty, a number of phenomenological models have been introduced to describe materials having limiting molecular chain extensibility, including the equivalent averaged stretch, full-network [4] and Arruda-Boyce 8-chain [5] models, the Gent [6], Puso [4], Treloar [7] models, and others [8-10], some that include a dependence on the second principal invariant [11]. These kinds of materials with limiting extensibility are collectively referred to as limited or restricted elastic materials.

Gent [6] has shown that the stiffening response in a simple tension predicted by his model is similar to that characterized by the classical molecular based Kuhn-Grün [1] statistical model in which the increasing stiffness arises from the inverse Langevin function. Both models exhibit rapidly increasing stresses as the stretch approaches its limiting value, though the Gent model does so at a somewhat smaller stretch. Boyce [12] has shown that when these models are compared with both uniaxial and biaxial experimental data by Treloar their physical response is virtually identical. It is important to mention that the averaged stretch model is widely known as the Arruda-Boyce 8chain model [5]. This constitutive equation was first reported by Wang and Guth [13, Equation (4.13b)] based on a 4-chain model, but they never explored it further. Four decades later, the relevance and easy derivation of the result for a simple 8-chain molecular based physical model was developed and studied by Arruda and Boyce [5] in comparison with experiments. Beatty [4] subsequently derived this result by approximation from the formidable constitutive equation for the amorphous, full- network molecular based Wu and van der Giessen model [14]. Therefore, emphasis on the special heuristic 8-cell morphology of the Arruda-Boyce construction is not necessary - the end result in [4] is simply the Arruda-Boyce constitutive equation for an averaged stretch, full-network of arbitrarily oriented molecular chains. All of the statistical mechanical models that are based on the Kuhn-Grün [1] probability distribution function, or its amended form discussed in [8], essentially exhibit dependence on only the first principal invariant of the Cauchy-Green deformation tensor. Consequently, the non-Gaussian molecular network models are characterized by a general constitutive equation whose response function depends on only the first principal invariant, and a certain molecular based material constant that characterizes its limiting extensibility.

In an effort to provide a molecular foundation for the material constants of the phenomenological Gent model, Horgan and Saccomandi [15] show analytically that when these constants are identified with those of the molecular based averaged stretch, fullnetwork model (that is, the Arruda-Boyce constitutive equation), the response function of the much simpler Gent model provides a very accurate approximation of the response function of the latter. In addition, they show that none of the appropriate Padé approximations $[16,17]$ of the inverse Langevin function of the molecular based model exhibit the correct singularity at the point of limiting extensibility, so they find no direct connection of the Gent model as a Padé approximant of the averaged stretch model. On the other hand, they demonstrate very close agreement of the Gent model with a certain modified Padé approximant due to Cohen [18]. Although it is true that the Gent model, as remarked in [15], cannot be obtained by a truncated power series approxima- 
tion of the molecular models based on the inverse Langevin function, this fact does not preclude its representation as an infinite power series with the correct limiting singularity, as described below.

The response function for a general but simple subclass of elastic molecular based materials characterized by their limiting molecular chain extensibility and depending on only the first principal invariant of the Cauchy-Green deformation tensor and a certain limiting extensibility parameter is introduced in Section 2 . The constitutive response function for the Gent material is then derived inversely in Section 3 as the [0/1] Padé approximant of this subclass of limited elastic materials, a result that leads naturally to an infinite geometric series representation of the Gent constitutive response function. Truncation of this series function characterizes a class of quadratic materials having physically relevant material constants, a model used frequently by others in nonlinear, finite amplitude vibration studies [19-23]. The subclass of response functions for limited elastic materials is expanded in Section 5 to characterize all limited elastic material models with dependence on the first principal invariant alone. These include the Arruda-Boyce, Cohen, Puso, Treloar, van der Waals and other restricted elastic materials. Certain Padé approximants of the averaged stretch model are not includedthese fail to exhibit the correct limiting chain singularity in the response, so these are not restricted elastic materials. Empirical modifications that render their membership in a few cases are described. It is shown that the [0/2] Padé approximant of the response function for the general class of restricted elastic materials leads inversely to a new constitutive model and its convergent series representation. The strain energy functions for a few limited elastic models, one being new, the others seldom seen, are presented at the end.

\section{Materials With Limiting Extensibility}

The theory of highly elastic, ideal rubberlike materials assumes that the range of the principal stretches $\lambda_{k}$ in any finite deformation may vary from zero to infinity: $0<\lambda_{k}<$ $\infty$, unless otherwise constrained. Of course, real rubberlike materials cannot be compressed or extended indefinitely, and various molecular based $[1,2,13,14]$ and phenomenological constitutive models $[4-6,8-11]$ have been introduced to characterize their limiting extensibility. This kinematical property is variously exhibited through the material response functions $\beta_{1}\left(I_{1}, I_{2}\right)$ and $\beta_{-1}\left(I_{1}, I_{2}\right)$ in the constitutive equation relating the Cauchy stress $\mathrm{T}$ to the Cauchy-Green deformation tensor B for a homogeneous, isotropic and incompressible elastic material, namely

$$
\mathbf{T}=-p \mathbf{1}+\beta_{1}\left(I_{1}, I_{2}\right) \mathbf{B}+\beta_{-1}\left(I_{1}, I_{2}\right) \mathbf{B}^{-1} .
$$

Here $I_{1}$ and $I_{2}$ are principal invariants of $\mathbf{B}, p$ is an arbitrary hydrostatic pressure, $\mathbf{1}$ is the identity tensor, and the response functions are required to satisfy the empirical inequalities

$$
\beta_{1}\left(I_{1}, I_{2}\right)>0, \quad \beta_{-1}\left(I_{1}, I_{2}\right) \leq 0
$$

for all deformations from an undistorted state of the material [24]. For hyperelastic materials with a strain energy function $\Sigma\left(I_{1}, I_{2}\right)$ per unit volume, the response functions are given by 


$$
\beta_{1}=2 \frac{\partial \Sigma}{\partial I_{1}}, \quad \beta_{-1}=-2 \frac{\partial \Sigma}{\partial I_{2}} .
$$

In terms of the principal stretches $\lambda_{k}$, we recall that the principal invariants for an incompressible material may be written as

$$
I_{1}=\lambda_{1}^{2}+\lambda_{2}^{2}+\lambda_{3}^{2}, \quad I_{2}=\lambda_{1}^{-2}+\lambda_{2}^{-2}+\lambda_{3}^{-2}, \quad I_{3}=\lambda_{1}^{2} \lambda_{2}^{2} \lambda_{3}^{2}=1,
$$

the last relation reflecting the incompressibility constraint.

Here we consider the class of molecular based constitutive models characterized by their limiting molecular chain extensibility so that any isochoric deformation of the material is restricted by imposing bounds on the extent of its deformation. Two kinds of bounds have been investigated. One method (see [9, 25], for example) restricts the greater of the three principal stretches, say $\lambda_{k}<\lambda_{m}$, where $\lambda_{m}$ is a certain kinematical material constant reflecting the limiting chain extensibility. In the molecular theory $\lambda_{m}$ $=\sqrt{N}$ is identified as the network locking constant, the limiting stretch of a chain of $N$ links [2]. A second method (see [4, 11], for example) imposes an upper bound on the first principal invariant, $I_{1}<I_{m}$, where $I_{m}$ is a certain kinematical material constant. This is consistent with results of molecular based theory in which $I_{m}=3 \mathrm{~N}$, for example $[4,5]$. But there are some difficulties associated with relating these methods (see $[4,8,25,26]$, for example).

These difficulties aside for now, we recall that experiments by Dickie and Smith [3] in simple extension, pure shear and equibiaxial deformation show that the degree of limiting extensibility of the material varies with the type of deformation. The limiting extensibility in a pure shear is only slightly smaller than that in a simple extension, whereas in an equibiaxial extension it is generally about $30 \%$ smaller. And these observations concur with the use of $I_{m}$ as the limiting extensibility parameter. Moreover, this extensibility limit is consistent with the experimental data in model comparisons reported by Boyce [12]. Therefore, these few experiments, as emphasized by Gent [27], clearly demonstrate that the limiting extensibility of noncrystallizing elastomers under various large, homogeneous deformations is governed by $I_{m}$ alone in all affine network deformations of the material. While this does not preclude a dependence on $I_{2}$ in (2.1), an independent limit on $I_{2}$ as a material constant is at variance with these results.

Therefore, in general terms, let $I_{m}$ denote a molecular based, dimensionless material constant and consider the simple subclass $\mathcal{C}_{0}$ of elastic, limiting extensibility materials defined by

$$
\beta_{1}\left(I_{1}, I_{2}\right)=G\left(I_{1} ; I_{m}\right), \quad G\left(3 ; I_{m}\right)=G_{0}>0, \quad \beta_{-1}\left(I_{1}, I_{2}\right) \equiv 0,
$$

such that the shear response function $G\left(I_{1} ; I_{m}\right) \geq G_{0}$ is a monotonic increasing function of $I_{1} \in\left[3, I_{m}\right]$ that grows indefinitely large as $I_{1} \rightarrow I_{m}$ for all deformations of the material:

$$
\lim _{I_{1} \rightarrow I_{m}} G\left(I_{1} ; I_{m}\right)=\infty
$$

Obviously, the empirical inequalities are satisfied. It is convenient to refer to all limiting chain extensibility models as limited or restricted elastic materials. To be specific, we 
recall below two models widely used in the literature for which $\beta_{-1} \equiv 0$ and $G_{0}$ denotes a material constant, usually the shear modulus of the undistorted, natural state. We are going to see that one limiting extensibility model satisfies both (2.5) and (2.6), the other does not. This leads subsequently to an extension of the definition (2.5). For our immediate purposes and for the sake of simplicity, however, it is convenient to begin with (2.5) and (2.6).

\section{Padé Approximants of the Response Function}

An $[L / M]$ Padé approximant $[16,17]$ is a particular type of rational fraction approximation of the value of a function $F(x)$ expressed as the ratio of two polynomials $P_{L}(x)=\sum$ ${ }_{i=0}^{L} a_{i} x^{i}$ of degree at most $L$ and $Q_{M}(x)=\sum_{j=0}^{M} b_{j} x^{j}$ of degree at most $M$. The idea usually is to match this ratio with a given power series expansion $F(x)=\sum_{\mathrm{k}=0}^{\infty} c_{k} x^{k}$ as closely as possible, but in simpler form so that, with only a few terms,

$$
F(x)-\frac{P_{L}(x)}{Q_{M}(x)}=\mathrm{O}\left(x^{L+M+1}\right) .
$$

The power series $F(x)$ thus determines the coefficients $a_{i}$ and $b_{j}$ upon solving a linear algebraic system. And the resultant [L/M] Padé approximant of $F(x)$ is unique. Here, however, we proceed differently, actually somewhat inversely.

We have in mind that the response function (2.5) is a certain power series whose precise form we wish not to specify at this time. We shall return to this momentarily. First, let us suppose that the response function $(2.5)_{1}$ is given by the simplest [0/1] Padé approximant, namely

$$
G\left(I_{1} ; I_{m}\right)=\frac{a_{0}}{b_{0}+b_{1} I_{1}},
$$

where $a_{0}, b_{0}, b_{1}$ are constants and $I_{1} \in\left[3, I_{m}\right]$. Since the ratio function is unaltered by the multiplication of both the numerator and the denominator by an arbitrary constant, there is no loss of generality in our setting $b_{0}=1$. We then find, in accordance with $(2.5)_{2}$, that $a_{0}=G_{0}\left(1+3 b_{1}\right)$; and, from the singularity condition (2.6), we take 1 $+b_{1} I_{m}=0$. Therefore, the simplest possible rational estimate (3.2) of the general response function having the properties $(2.5)_{2}$ and (2.6) is given by the monotonic increasing function

$$
\beta_{1}=G\left(I_{1} ; I_{m}\right)=G_{0}\left(\frac{1-3 / I_{m}}{1-I_{1} / I_{m}}\right) .
$$

This is exactly the response function for the phenomenological Gent material [6], often written as

$$
\beta_{1}=G\left(I_{1} ; I_{m}\right)=G_{0}\left(1-\frac{I_{1}-3}{I_{m}-3}\right)^{-1}
$$


Though Pucci and Saccomandi [11] essentially recognized that the Gent response function is an [0/1] rational approximation of what they called the reduced tensile force, they did not derive it inversely from the general properties of the simple class $\mathcal{C}_{0}$. In a different, general framework, Horgan and Saccomandi [28] apply a modified Rivlin polynomial approximation scheme in which the strain energy function is derived by integration of (2.3) for assumed rational function approximants of the response functions that may involve both principal invariants for an incompressible hyperelastic material. In addition, they obtain a thermoelastic Gent model by the extension of their procedure to thermoelastic materials with an additional thermal invariant dependence. The Gent constitutive equation (3.3) or (3.4), however, here follows easily and naturally from only the fundamental properties of general limited elastic materials without mention of a strain energy function. We shall return to this farther on.

Now consider our second restricted elastic model. The response function for the molecular based, averaged stretch, full-network model [4], also widely known as the Arruda-Boyce 8-chain model [5], is described by

$$
\beta_{1}=G\left(I_{1} ; I_{m}\right)=G_{0} \frac{\mathcal{L}^{-1}\left(\lambda_{r}\right)}{3 \lambda_{r}},
$$

in which $\mathcal{L}^{-1}\left(\lambda_{r}\right)$ is the inverse of the Langevin function

$$
\lambda_{r}=\mathcal{L}(\beta) \equiv \operatorname{coth} \beta-1 / \beta
$$

and $\lambda_{r} \equiv \sqrt{I_{1} / I_{m}}$ is called the relative chain stretch. The Taylor series approximation [7, p. 895] of $\mathcal{L}^{-1}\left(\lambda_{r}\right)$ derived from (3.6) is given by

$$
\mathcal{L}^{-1}\left(\lambda_{r}\right)=3 \lambda_{r}+\frac{9}{5} \lambda_{r}^{3}+\frac{297}{175} \lambda_{r}^{5}+\frac{1539}{875} \lambda_{r}^{7}+\frac{126117}{67375} \lambda_{r}^{9}+\cdots
$$

Based on this series expansion, Cohen [18] has derived the [3/2] Padé approximant of the inverse Langevin function; and introduction of this result in the response function (3.5) leads to

$$
G\left(I_{1} ; I_{m}\right)=G_{0} \frac{1-\frac{12}{35} \lambda_{r}^{2}}{1-\frac{33}{35} \lambda_{r}^{2}}+\mathrm{O}\left(\lambda_{r}^{5}\right) \approx \frac{G_{0}}{3} \frac{3-\lambda_{r}^{2}}{1-\lambda_{r}^{2}}
$$

The latter approximate expression is adopted by Cohen to capture exactly the singularity of $\mathcal{L}^{-1}\left(\lambda_{r}\right)$ for large chain stretches at $\lambda_{r}=1$, for which the approximant (3.8) fails. Similarly, the [1/6] Padé approximant [15] of (3.7) is given by

$$
\mathcal{L}^{-1}\left(\lambda_{r}\right)=\frac{3 \lambda_{r}}{1-\left(\frac{3}{5} \lambda_{r}^{2}+\frac{36}{175} \lambda_{r}^{4}+\frac{108}{875} \lambda_{r}^{6}\right)} \approx \frac{3 \lambda_{r}}{1-\left(\frac{3}{5} \lambda_{r}^{2}+\frac{1}{5} \lambda_{r}^{4}+\frac{1}{5} \lambda_{r}^{6}\right)}
$$


The first of these relations was obtained initially by Kuhn and Grün [1] based on (3.7), but not by the method of Padé approximants. More than a decade later, Treloar [7] introduced the latter empirical estimate $(3.9)_{2}$ to preserve the singularity at $\lambda_{r}=1$, for which the [1/6] approximant (3.9) fails. Treloar's estimate $(3.9)_{2}$ is within $1 \%$ of the exact value of $\mathcal{L}^{-1}\left(\lambda_{r}\right)$ over the entire range $\lambda_{r}$. Notice, however, that the response function (3.5) and hence those for the modified Cohen and Treloar functions (3.8)2 and (3.9)2 are not members of the simple subclass $\mathcal{C}_{0}$ of limited elastic materials; they do not satisfy $(2.5)_{2}$. We shall return to this below.

With (3.3) or (3.4) cast in these molecular based terms the analytical simplicity of the Gent model in comparison with (3.5), or even the Cohen and Treloar empirical approximations, is evident:

$$
\beta_{1}=G_{0} \frac{1-3 / I_{m}}{1-\lambda_{r}^{2}}
$$

The Gent model, therefore, is the simplest rational function of $\lambda_{r}$ having the properties (2.5) and (2.6). This is not, as shown in [15], an [0/2] Padé approximant of the ratio function in (3.5), which is given by

$$
\frac{\mathcal{L}^{-1}\left(\lambda_{r}\right)}{3 \lambda_{r}}=\frac{1}{\left(1-3 \lambda_{r}^{2} / 5\right)}+\mathrm{O}\left(\lambda_{r}^{3}\right)
$$

This has the correct quadratic structure of (3.10) but it does not exhibit the required singularity at $\lambda_{r}=1$.

\section{Series Representations of the Gent Model}

Now let us return to the Gent model, and recall that $0 \leq\left(I_{1}-3\right) /\left(I_{m}-3\right) \leq 1$, the equalities holding only at the respective end points $I_{1}=3$ and $I_{1}=I_{m}$. It is readily shown that the Gent response function (3.4) has an infinite geometric series [29, pp. 111-112] representation that converges absolutely and uniformly to precisely the Gent response function for $\left(I_{1}-3\right) /\left(I_{m}-3\right)<1$, namely

$$
G\left(I_{1} ; I_{m}\right)=G_{0} \sum_{n=0}^{\infty}\left(\frac{I_{1}-3}{I_{m}-3}\right)^{n}=G_{0} \frac{1}{1-\frac{I_{1}-3}{I_{m}-3}}
$$

An alternate series representation based on (3.3) is

$$
G\left(I_{1} ; I_{m}\right)=G_{0}\left(1-\frac{3}{I_{m}}\right) \sum_{n=0}^{\infty}\left(\frac{I_{1}}{I_{m}}\right)^{n}=G_{0}\left(\frac{1-3 / I_{m}}{1-I_{1} / I_{m}}\right)
$$


in which we note that $\sum_{n=0}^{\infty}(3 / I m)^{n}=\left(1-3 / I_{m}\right)^{-1}$. These relations, therefore, are merely alternate forms of the infinite geometric series for a positive quantity $k<1$, namely, $\sum_{n=0}^{\infty} k^{n}=(1-k)^{-1}$, and this is the fundamental basis for the Gent or any similar model, an example of which is provided later on.

Notice that the series functions $(4.1)_{1}$ and (4.2), satisfy (2.5) 2 when $I_{1}=3$ and (2.6) for which $\sum_{n=0}^{\infty} 1^{n}=\infty$ when $I_{1}=I_{m}$, but any truncation of either series at a finite number of terms, like the series (3.7), does not. In particular, consider the function (4.1) for $n=1$ to obtain

$$
G\left(I_{1} ; I_{m}\right)=G_{0}\left(1+\frac{I_{1}-3}{I_{m}-3}\right) .
$$

Let us now recall a simple shear deformation [24] with an amount of shear $\sigma=\tan \alpha$, where $\alpha$ is the angle of shear, and $I_{1}-3=\sigma^{2}$. Let $\sigma_{m}$ denote the limiting amount of shear for which $I_{m}-3=\sigma_{m}^{2}$. Then (4.3) may be written as

$$
\mu\left(\sigma^{2}\right)=\mu_{0}+2 \mu_{1} \sigma^{2}
$$

in which $\mu\left(\sigma^{2}\right)=G\left(\sigma^{2}+3 ; I_{m}\right), \mu_{0}=G_{0}$, and $2 \mu_{1}=G_{0} / \sigma_{m}^{2}$. The problem of the vibration of a load supported symmetrically by simple shear springs was solved exactly in [19] for all isotropic hyperelastic materials characterized by the quadratic shear response function (4.4); and this simple shear problem has served as a signature example in the study of a variety of analytical methods applied to nonlinear vibration problems [19-23]. Here we find a specific representation for the moduli in (4.4) in terms of meaningful physical constants whose connection with the Gent model was previously unknown. An exact solution of the shearing oscillator problem for the Gent model (3.3) is reported in [30] where several additional references on shearing oscillation problems may be found.

\section{Extended Class of Limited Elastic Materials}

It is known that the function (3.5) has only the property (2.6). Hence, the averaged stretch, full-network model, which clearly exhibits limited extensibility as $\lambda_{r} \rightarrow 1$, is not a member of the class $\mathcal{C}_{0}$. For the averaged stretch model, the value of the response function $G\left(3 ; I_{m}\right)=G_{0} \sqrt{I_{m} / 3} \mathcal{L}^{-1}\left(\sqrt{3 / I_{m}}\right) / 3$ depends on the material extensibility constant $I_{m}$. So, $(2.5)_{2}$ holds only for sufficiently small $\lambda_{r}$, that is, when $I_{m}$ is sufficiently large; and then $G\left(3 ; I_{m}\right) \rightarrow G_{0}$. Otherwise, the material constant $G\left(3 ; I_{m}\right)$, the response modulus of the undeformed state for the averaged stretch model, also depends on the material constant $I_{m}$. This suggests extension of the definition (2.5) to the general class $\mathcal{C}$ of limiting elastic materials defined by

$$
G\left(3 ; I_{m}\right)=\mathcal{G}\left(I_{m}\right) \text { such that } \lim _{I_{m} \rightarrow \infty} \mathcal{G}\left(I_{m}\right)=G_{0}>0
$$

together with (2.6), where $\mathcal{G}\left(I_{m}\right)$, the shear modulus in the undeformed state of the material, is a positive-valued function of the extensibility constant $I_{m}$. The models for 
which (2.5) hold clearly form a subclass of those defined by (5.1) and for which it is always admissible to consider a model, such as the Gent model and another to be discussed shortly, for which $G\left(3 ; I_{m}\right)=\mathcal{G}\left(I_{m}\right) \equiv G_{0}$.

Various other models for which $(2.5)_{2}$ fails, including the modified Cohen model $(3.8)_{2}$ and the Treloar model (3.9)2, are now included in the expanded class $\mathcal{C}$ of limited elastic materials for which (2.6) and (5.1) hold. An alternative but less elegant approach is to modify the material response function (3.5) to remove the $I_{m}$ dependence by an evident normalization so that both (2.5) and (2.6) hold, which then leads to a somewhat different material model. Notice that the location of the singularity for the Padé approximants (3.8)1, (3.9)1, and (3.11) fall outside the range of limiting molecular chain extensibility at $\lambda_{r}=1$, so these are not limited elastic material models. Clearly, various limited elastic models having a response function defined in terms of powers of principal stretches or additional invariants are not members of the class studied here. Needless to say, there are several limited elastic or modified limited elastic models, such as the van der Waals model [15] with

$$
G\left(I_{1} ; I_{m}\right)=G_{0}\left(1-\sqrt{\frac{I_{1}-3}{I_{m}-3}}\right)^{-1}
$$

for which (2.5), hence (5.1), and (2.6) hold; and the Puso model [4] defined by

$$
G\left(I_{1} ; I_{m}\right)=G_{0}\left(1-\left(\frac{I_{1}}{I_{m}}\right)^{3 / 2}\right)^{-1}
$$

for which $G\left(3 ; I_{m}\right)=\mathcal{G}\left(I_{m}\right)=G_{0} /\left(1-\left(3 / I_{m}\right)^{3 / 2}\right)$ and hence (5.1) and (2.6) hold. Similarly, it is seen that the empirical Treloar $\left[2\right.$, p. 17817, p. 895] model $(3.9)_{2}$ with

$$
G\left(I_{1} ; I_{m}\right)=G_{0} \frac{1}{1-\phi\left(\lambda_{r}^{2}\right)},
$$

in which $\phi\left(\lambda_{r}^{2}\right)=3 \lambda_{r}^{2} / 5+\lambda_{r}^{4} / 5+\lambda_{r}^{6} / 5$, and $0<\phi\left(\lambda_{r}^{2}\right) \leq 1$, equality holding at $\lambda_{r}=1$, is a member of the extended class $\mathcal{C}$ of limited elastic materials for which (5.1) and (2.6) hold. None of these additional models, however, are Padé approximants. In fact, the van der Waals and Puso models are not rational fractions, and the Cohen and Treloar empirical models are rounded estimates of Padé approximants of the inverse Langevin function modified to exhibit the correct singularity at $\lambda_{r}=1$. Notice, however, that several of our models in the class $\mathcal{C}$ have essentially the same structure, which suggests that these may be represented by their infinite geometric series in $a=f\left(I_{1} ; I_{m}\right)$, for $0<a<1$, that sums exactly to the simplified form $\left(1-f\left(I_{1} ; I_{m}\right)^{-1}\right.$. The van der Waals model (5.2), for example, may be written as an infinite geometric series in $a=f\left(I_{1} ; I_{m}\right)=\left[\left(I_{1}-3\right) /\left(I_{m}\right.\right.$ $-3)]^{1 / 2}$, where $0<\left(I_{1}-3\right) /\left(I_{m}-3\right)<1$. The Gent model, however, is unique among all of these - it is the only limited elastic material that is also the unique [0/1] Padé approximant of the general class of restricted elastic materials characterized by (2.5) and (2.6) and having the exact infinite geometric series representation (4.1) or (4.2). 
It is evident from the molecular theory that larger values of the extensibility parameter $I_{m}$ imply greater elastic (extensibility) response of the material, while smaller values of $I_{m}$ imply its greater stiffness. Hence, as $I_{m}$ grows indefinitely large, (3.3) for the Gent material shows that $\beta_{1} \rightarrow G_{0}$ and (2.1) thus reduces to the constitutive equation for the classical, ideal elastic neo-Hookean model of the Gaussian molecular network theory. For all materials within the class $\mathcal{C}$ of limiting extensibility materials defined by (5.1) and (2.6), this reduction to the neo-Hookean case may be generalized by the additional condition that

$$
\lim _{I_{m} \rightarrow \infty} G\left(I_{1} ; I_{m}\right)=G_{0}
$$

and in some applications it is occasionally useful to include

$$
\lim _{I_{m \rightarrow \infty}} \partial G\left(I_{1} ; I_{m}\right) / \partial I_{1}=0
$$

Both the Gent and averaged stretch, full-network models respect these additional properties. For the latter, we recall $\lambda_{r} \in\left[3 / I_{m}, 1\right]$, and note that $\lim _{\lambda_{r} \rightarrow 1} \mathcal{L}^{-1}\left(\lambda_{r}\right)=\lim _{I_{1} \rightarrow I_{m}} \mathcal{L}^{-}$ ${ }^{1}\left(\lambda_{r}\right) \rightarrow \infty$; and as $I_{m} \rightarrow \infty$, the ratio in (3.5) goes to 1 .

\section{A New Restricted Elastic Model}

We have seen that the Gent model is not the [0/2] Padé approximant (3.11) of the ratio function in (3.5), which has the correct quadratic structure but does not exhibit the required singularity at $\lambda_{r}=1$. Let us return to our general model defined by (2.5) and (2.6), and let us consider the [0/2] Padé approximant for which

$$
G\left(I_{1} ; I_{m}\right)=\frac{a_{0}}{1+b_{1} I_{1}+b_{2} I_{1}^{2}}
$$

To simplify the calculation, set $a_{0}=G_{0}$, that is, we chose $\mathcal{G}\left(I_{m}\right)=G_{0}$ in (5.1). Then to satisfy $(5.1)_{2}$, or equivalently $(2.5)_{2}$, we must have $b_{1}+3 b_{2}=0$; and to satisfy (2.6) requires 1 $+b_{1} I_{m}+b_{2} I_{m}^{2}=0$. Solving this algebraic system for $b_{1}$ and $b_{2}$, we find that the [0/2] Pade approximant of the general response function for which (6.1) holds may be written as

$$
G\left(I_{1} ; I_{m}\right)=G_{0} \frac{1}{1-\frac{I_{1}}{I_{m}}\left(\frac{I_{1}-3}{I_{m}-3}\right)}
$$

In addition, this function also satisfies (5.5) and (5.6), and it has the exact infinite geometric series representation

$$
G\left(I_{1} ; I_{m}\right)=G_{0} \sum_{n=0}^{\infty} a^{n}=G_{0} \frac{1}{1-a}, \quad a \equiv \frac{I_{1}}{I_{m}}\left(\frac{I_{1}-3}{I_{m}-3}\right),
$$


for $0<a<1$. Notice that for $I_{1}=3, a=0$ and $(2.5)_{2}$ holds, and when $I_{1}=I_{m}, \sum_{n=0}^{\infty} a^{n} \rightarrow+\infty$ and (2.6) holds. This is a new constitutive equation for incompressible, isotropic limited elastic materials.

\section{Strain Energy of Restricted Elastic Materials}

We have seen that the constitutive equations for the Gent material (3.4) and the new relation (6.2) follow easily and naturally from the fundamental properties of the general class of limited elastic materials without mention of a stored energy function. Of course, this function is readily obtained by integration of (2.3) $)_{1}$. Gent's strain energy function [6] follows easily from (3.4):

$$
\Sigma\left(I_{1} ; I_{m}\right)=-\frac{G_{0}}{2}\left(I_{m}-3\right) \log \left(1-\frac{I_{1}-3}{I_{m}-3}\right) .
$$

And the strain energy function for the new elastic material (6.2) is given by

$$
\Sigma\left(I_{1} ; I_{m}\right)=-\frac{G_{0} I_{m}\left(I_{m}-3\right)}{2\left(2 I_{m}-3\right)} \log \left(\frac{1-\frac{I_{1}-3}{I_{m}-3}}{1+\frac{I_{1}-3}{I_{m}}}\right) .
$$

The similarity with the much simpler Gent model is evident.

The stored energy function for the Puso model (5.3) is given by

$$
\begin{aligned}
\Sigma\left(I_{1} ; I_{m}\right) & =\frac{1}{6} G_{0} I_{m}\left(\ln \left(\frac{1+\sqrt{\frac{I_{1}}{I_{m}}}+\frac{I_{1}}{I_{m}}}{\left(1-\sqrt{\frac{I_{1}}{I_{m}}}\right)^{2}}\right)\right. \\
& \left.-2 \sqrt{3} \arctan \frac{1}{\sqrt{3}}\left(1+2 \sqrt{\frac{I_{1}}{I_{m}}}\right)\right)+C_{0},
\end{aligned}
$$

in which here and below the constant $C_{0}$ is chosen so that $\Sigma\left(3 ; I_{m}\right)=0$. This structure is similar to the empirical Treloar model (5.4) for which the strain energy function is given by

$$
\Sigma\left(I_{1} ; I_{m}\right)=\frac{5}{32} G_{0} I_{m}\left(\ln \left(\frac{4+\left(1+\frac{I_{1}}{I_{m}}\right)^{2}}{\left(1-\frac{I_{1}}{I_{m}}\right)^{2}}\right)+2 \arctan \frac{1}{2}\left(1+\frac{I_{1}}{I_{m}}\right)\right)+C_{0}
$$




$$
\Sigma\left(I_{1} ; I_{m}\right)=\frac{G_{0}}{2}\left(I_{1}-3\right)+\frac{G_{0}}{4\left(I_{m}-3\right)}\left(I_{1}-3\right)^{2},
$$

in terms of physical, molecular based material coefficients. This also follows by the series expansion of (7.1). This is a special variety within the general class of compressible and incompressible quadratic energy functions applied in [19] to study simple shear oscillators for which (4.4) holds. Now, however, the material coefficients have an explicit physical relevance in terms of the shear modulus G0 and the limiting extensibility parameter $I_{m}$.

For all of these models the $\lim _{I_{m \rightarrow \infty}} \Sigma\left(I_{1} ; I_{m}\right)=G_{0}\left(I_{1}-3\right) / 2$, the familiar neo-Hookean constitutive equation of the Gaussian molecular network theory. Plainly, the functions (7.1)-(7.4) have infinite power series representations for $3 \leq I_{1}<I_{m}$. So, specifically, it may be shown, though it most surely is not necessary to do so, that the result for (7.1) follows from the infinite geometric series (5.5), and conversely. Indeed, in consequence of the logarithmic functions in (7.1) and (7.2), for example, it is not surprising that the response functions for these models should have the respective infinite power series representations (4.1) and (6.3). Indeed, for $0<x<1$, we have exactly

$$
\frac{\mathrm{d}}{\mathrm{d} x} \log (1-x)=-\frac{\mathrm{d}}{\mathrm{d} x} \sum_{n=1}^{\infty} \frac{x^{n}}{n}=-\sum_{m=0}^{\infty} x^{m}=-\frac{1}{1-x} .
$$

\section{References}

[1] Kuhn, W. and Grün, F. Beziehungen zwischen elastischen Konstanten und Dehnungsdoppelbrechung hochelastischer Stoffe. Kolloid-Zeitschrift, 101, 248-271 (1942).

[2] Treloar, L. R. G. The Physics of Rubber Elasticity, 3rd edn. Clarendon Press, Oxford, 1975.

[3] Dickie, R. A. and Smith, T. L. Viscoelastic properties of a rubber vulcanizate under large deformations in equal biaxial tension, pure shear, and simple tension. Transactions of the Society of Rheology, 15, 91-110 (1971).

[4] Beatty, M. F. A stretch averaged full-network model for rubber elasticity. Journal of Elasticity, 70, 65-86 (2003).

[5] Arruda, E. M. and Boyce, M. C. A three-dimensional constitutive model for the large stretch behavior of rubber elastic materials. Journal of the Mechanics and Physics of Solids, 41, 389-412 (1993).

[6] Gent, A. N. A new constitutive relation for rubber. Rubber Chemistry and Technology, 69, 59-61 (1996).

[7] Treloar, L. R. G. The photoelastic properties of short-chain molecular networks. Transactions of the Faraday Society, 50, 881-896 (1954).

[8] Zúñiga, A. Elías-and Beatty, M. F. Constitutive equations for amended non-Gaussian network models of rubber elasticity. International Journal of Engineering Science, 40, 2265-2294 (2002).

[9] Horgan, C. O. and Saccomandi, G. Constitutive modelling of rubber-like and biological materials with limiting chain extensibility. Mathematics and Mechanics of Solids, 7, 353-371 (2002).

[10] Horgan, C. O. and Saccomandi, G. Phenomenological hyperelastic strain-stiffening constitutive models for rubber. Rubber Chemistry and Technology, 79, 152-169 (2006).

[11] Pucci, E. and Saccomandi, G. A note on the Gent model for rubber-like materials. Rubber Chemistry and Technology, 75, 839-851 (2002).

[12] Boyce, M. C. Direct comparison of the Gent and the Arruda-Boyce constitutive models of rubber elasticity. Rubber Chemistry and Technology, 69, 781-785 (1996). 
[13] Wang, M. C. and Guth, E. Statistical theory of networks of non-Gaussian flexible chains. Journal of Chemical Physics, 20, 1144-1157 (1952).

[14] Wu, P. D. and van der Giessen, E. On improved network models for rubber elasticity and their application to orientation hardening in glassy polymers. Journal of the Mechanics and Physics of Solids, 41, 427-456 (1993).

[15] Horgan, C. O. and Saccomandi, G. A molecular-statistical basis for the Gent constitutive model of rubber elasticity. Journal of Elasticity, 68, 167-176 (2002).

[16] Baker, G. A. Essentials of Padé Approximants, Academic, New York, 1975.

[17] Cabannes, H. Padé Approximants Method and Its Applications to Mechanics, Vol. 47, Lecture Notes in Physics, Springer, Berlin, 1976.

[18] Cohen, A. A Padé approximant to the inverse Langevin function. Rheologica Acta, 30, 270-273 (1991).

[19] Beatty, M. F. Finite amplitude vibrations of a body supported by simple shear springs. Journal of Applied Mechanics, 51, 361-366 (1984).

[20] Beatty, M. F. and Bhattacharyya, R. Stability of the free vibrational motion of a vehicular body supported by rubber shear mountings with quadratic response. International Journal of NonLinear Mechanics, 24, 401-414 (1989).

[21] Zúñiga, A. Elias-. Absorber control of the $\mathrm{f}$ inite amplitude nonlinear vibrations of a simple shear suspension system. University of Nebraska-Lincoln, Ph.D. dissertation, 1994.

[22] Zúñiga, A. Elias- and Beatty, M. F. Forced vibrations of a body supported by hyperelastic shear mountings. Mechanics Research Communications, 28, 429-446 (2001).

[23] Zúñiga, A. Elias-and Beatty, M. F. Elliptic balance solution of two degree-of-freedom, undamped, forced systems with cubic nonlinearity, Nonlinear Dynamics, in press.

[24] Beatty, M. F. Introduction to nonlinear elasticity, in Nonlinear Effects in Fluids and Solids, dedicated to Ronald S. Rivlin, eds. M. M. Carroll and M. A. Hayes, Plenum Press, New York, 1996, pp. 13-112. Alternatively, see Topics in finite elasticity: Hyperelasticity of rubber, elastomers, and biological tissues - with examples. Applied Mechanics Reviews, 40, 1699-1734 (1987).

[25] Carroll, M. M. Polymers with limiting chain extensibility. Private communication.

[26] Murphy, J. G. Some remarks on kinematic modeling of limiting chain extensibility. Mathematics and Mechanics of Solids, 11, 629-641 (2006).

[27] Gent, A. N. Extensibility of rubber under different types of deformation. Journal of Rheology, 49, 271-275 (2005).

[28] Horgan, C.O. and Saccomandi, G., Finite thermoelasticity with limiting chain extensibility. Journal of the Mechanics and Physics of Solids, 51, 1127-1146 (2003).

[29] Knopp, K. Theory and Application of Infinite Series, a translation by R. C. H. Young of the 4th German ed., Hafner, New York, 1947.

[30] Beatty, M. F. On the oscillations of a load supported by incompressible, isotropic limited elastic shear mounts (in press). 